BIOMEDICAL AND BIOSOCIAL ANTHROPOLOGY
$\begin{gathered}\text { Official Journal of the International Academy } \\ \text { of Integrative Anthropology } \\ \text { journal homepage: http://bba-journal.com }\end{gathered}$

\title{
Evaluation of changes in instrumental and biochemical markers of heart diseases in patients with bladder cancer under the condition of intravenous and intravesical doxorubicin administration
}

\section{Hodovan N. L.}

National Pirogov Memorial Medical University, Vinnytsya, Ukraine

\section{ARTICLE INFO \\ Received: 22 March 2021 \\ Accepted: 23 April 2021}

UDC: $616.62-006.5+616-$

$006.6+616.31-07$

\section{CORRESPONDING AUTHOR}

e-mail: sciencepharmak@gmail.com Hodovan N. L.

\begin{abstract}
Due to the constant increase in morbidity and mortality, bladder cancer remains a pressing problem in modern medicine. Despite the success of chemotherapy, chemotherapy physicians around the world have been concerned about the safety of these drugs for many years. Doxorubicin has been used as an antitumor drug for more than 40 years in various hematological and solid malignancies, however, its toxic effects when administered intravenously to vital organs and organ systems, including the heart, require further research. The aim of our study was to evaluate the manifestations and progression of cardiotoxicity in patients with bladder cancer with systemic and intravesical administration of doxorubicin. The study included 96 patients who were divided into three groups: the first group or the control group - patients who had a confirmed diagnosis of first stage bladder cancer, were treated surgically and did not receive doxorubicin; the second group - patients who, in addition to surgical treatment, received doxorubicin systemically (intravenously) in a therapeutic dose; the third group - patients who received doxorubicin at a dose of $50 \mathrm{mg}$, locally, by instillation of the drug into the bladder cavity, after prior catheterization with an elastic catheter. ECG data and biochemical markers of myocardial destruction before and after chemotherapy were studied. The study found that systemic serial administration of doxorubicin to patients with bladder cancer causes significant changes in the myocardium after the first course of chemotherapy. A single intravesical instillation of doxorubicin does not cause changes in laboratory and instrumental heart rate.

Keywords: bladder cancer, chemotherapy, myocardial destruction, doxorubicin.
\end{abstract}

\section{Introduction}

Due to the constant increase in morbidity and mortality, bladder cancer remains a pressing problem in modern medicine. Bladder cancer is the fourth most common cancer in the United States and Europe. In terms of mortality, this pathology ranks seventh and eighth in the structure of mortality from oncopathology in the United States [14] and Europe, respectively [4]. Tumors of the bladder occur 3-4 times more often in men than in women [14]. The overall five-year survival rate in Europe is about $68 \%$ [2].

The situation in Ukraine is not better. Thus, almost 5,000 new cases and more than 2,000 deaths from this pathology are registered annually [9].

Due to the constant improvement of diagnostic algorithms, more and more newly diagnosed cases (according to some data up to $75 \%$ ) are superficial bladder cancer, when the tumor affects only the mucous membrane or submucosal layer [1].

Despite the success of chemotherapy, chemotherapy physicians around the world have been concerned about the safety of these drugs for many years. The ambiguity of approaches to the treatment of cancer patients indicates the scale of the problem of optimal chemotherapy. In many countries around the world, cover drugs are used, but the pharmacological action of these drugs is aimed at protecting individual organs, and the number of drugs taken by the patient raises concerns about the pharmacokinetic relationships of these drugs and increasing toxicity when taken together. On the other hand, the search for certain methods of delivery of chemotherapeutics to the focus of the cancer process is a very promising area of oncology [7].

The standard of care for patients with superficial bladder cancer is transurethral resection of the tumor [20]. Systemic 
or, less frequently, intravesical chemotherapy and immunotherapy are used as adjuvant therapy after transurethral resection to prevent the development of residual and recurrent neoplasms $[13,22]$.

Doxorubicin is an anthracycline drug that has been used for more than 40 years as an antitumor drug in various hematological and solid malignancies [11]. However, its toxic effects when administered intravenously, on vital organs and organ systems puts doctors at a dead end. A particularly dangerous complication of doxorubicin chemotherapy is the development of cardiotoxicity $[11,17]$.

The aim of the study was to evaluate the manifestations and progression of cardiotoxicity in patients with bladder cancer with systemic and intravesical administration of doxorubicin.

\section{Materials and methods}

The prospective study was conducted at the Department of Radiation Diagnostics, Radiation Therapy and Oncology of National Pirogov Memorial Medical University, Vinnytsya on the basis of the Podolsk Regional Oncology Center.

The study was conducted with the permission of the Committee on Bioethics of National Pirogov Memorial Medical University, Vinnytsya and did not contradict the basic bioethical norms of the Helsinki Declaration adopted by the General Assembly of the World Medical Association, the Council of Europe Convention on Human Rights and Biomedicine (1977), the relevant provisions of the WHO, the International Council of Medical Societies, the International Code of Medical Ethics (1983).

A total of 96 patients with bladder cancer were examined as part of the study.

All patients were divided into three groups:

- first group or control group (35 people) - patients who had a confirmed diagnosis of first stage bladder cancer, were treated surgically and did not receive doxorubicin;

- the second group - 26 patients who, in addition to surgical treatment, received doxorubicin systemically (intravenously) in a therapeutic dose, which was calculated individually according to body surface area;

- third group - 35 patients who received doxorubicin at a dose of $50 \mathrm{mg}$, locally, by instillation of the drug into the bladder cavity, after preliminary catheterization with an elastic catheter.

All patients underwent ECG with baseline parameters and biochemical blood tests prior to and at the end of chemotherapy, including alanine aminotransferase (ALT), aspartate aminotransferase (AST), and creatine phosphokinase (CPK). The data were compared between groups of patients with different approaches to the treatment of bladder cancer. These studies were mandatory because their results determined the duration of the intervals between chemotherapy courses. The obtained data were processed using the statistical software package SPSS 20.0 for Windows. The difference in ALT, AST and CPK activity was considered significant at $\mathrm{p} \leq 0.05$.

\section{Results}

Our studies showed that the first group of patients who received only surgical treatment and did not receive doxorubicin, no anamnestic, laboratory or instrumental data that would indicate cardiovascular disorders were found.

At the same time, in the second group only a part of patients noted discomfort in work of heart, instead, at other patients any symptomatology was absent. Already in the first week, about $27 \%$ of patients showed signs of cardiovascular damage, but by the end of the 4th course, $47 \%$ of patients experienced pain in the heart and rapid

Table 1. The main parameters of the ECG in the studied contingent.

\begin{tabular}{|c|c|c|c|c|c|c|}
\hline \multirow[b]{2}{*}{ Terms of research } & \multicolumn{6}{|c|}{ ECG indicators } \\
\hline & $\begin{array}{l}\text { Heart rate, } \\
\text { beats/min }\end{array}$ & $\mathrm{P}, \mathrm{s}$ & $\mathrm{R}-\mathrm{R}, \mathrm{s}$ & S-T, s & QRS, s & QT, s \\
\hline \multicolumn{7}{|c|}{ First group (Control group) } \\
\hline At the beginning of treatment & $71.74 \pm 9.262$ & $0.111 \pm 0.119$ & $0.845 \pm 0.108$ & $0.149 \pm 0.144$ & $0.106 \pm 0.013$ & $0.392 \pm 0.025$ \\
\hline At the end of treatment & $75.45 \pm 10.96$ & $0.097 \pm 0.017$ & $0.807 \pm 0.115$ & $0.120 \pm 0.025$ & $0.106 \pm 0.012$ & $0.386 \pm 0.029$ \\
\hline \multicolumn{7}{|c|}{ The second group - Systemic administration of doxorubicin } \\
\hline 1 course & $68.19 \pm 7.082$ & $0.099 \pm 0.102$ & $0.774 \pm 0.218$ & $0.123 \pm 0.027$ & $0.102 \pm 0.011$ & $0.386 \pm 0.031$ \\
\hline 2 course & $75.03 \pm 9.848$ & $0.096 \pm 0.015$ & $0.780 \pm 0.122$ & $0.109 \pm 0.025$ & $0.104 \pm 0,012$ & $0.372 \pm 0.026$ \\
\hline 3 course & $76.92 \pm 10.47$ & $0.0971 \pm 0.017$ & $0.754 \pm 0.161$ & $0.118 \pm 0.025$ & $0.106 \pm 0.014$ & $0.385 \pm 0.020$ \\
\hline 4 course & $77.46 \pm 11.02$ & $0.101 \pm 0.017$ & $0.744 \pm 0.204$ & $0.119 \pm 0.022$ & $0.111 \pm 0.020$ & $0.391 \pm 0.019$ \\
\hline 5 course & $85.84 \pm^{*} 12.63$ & $0.102 \pm 0.022$ & $0.746 \pm 0.216$ & $0.118 \pm 0.028$ & $0.166 \pm 0.027$ & $0.392 \pm 0.029$ \\
\hline \multicolumn{7}{|c|}{ The third group - Intravesical administration } \\
\hline At the beginning of treatment & $74.84 \pm 14.19$ & $0.090 \pm 0.027$ & $0.660 \pm 0.080$ & $0.096 \pm 0.020$ & $0.091 \pm 0.008$ & $0.392 \pm 0.040$ \\
\hline At the end of treatment & $76.00 \pm 8.420$ & $0.099 \pm 0.017$ & $0.754 \pm 0.070$ & $0.078 \pm 0.038$ & $0.097 \pm 0.007$ & $0.371 \pm 0.031$ \\
\hline
\end{tabular}

Note: ${ }^{*}$ - the difference is significant compared to the control group. 


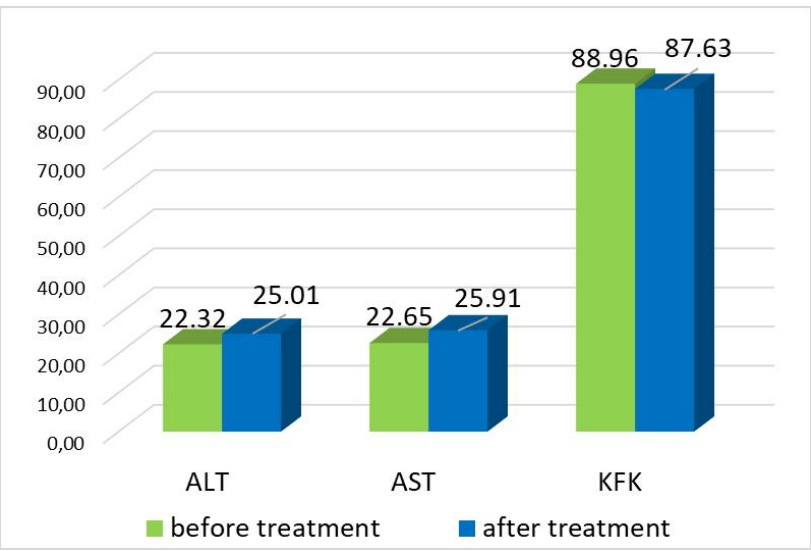

Fig. 1. Dynamics of biochemical parameters of myocardial destruction in patients not receiving doxorubicin.

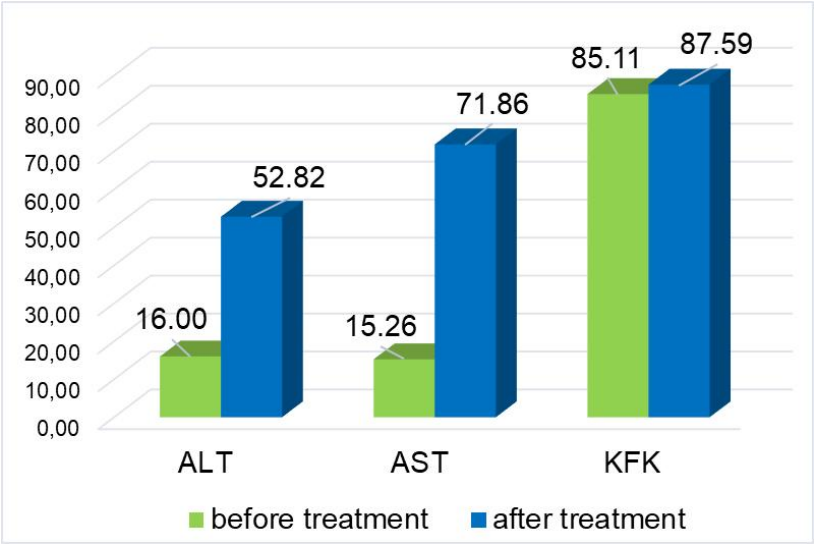

Fig. 2. Dynamics of biochemical parameters of myocardial destruction in patients prescribed intravenous doxorubicin.

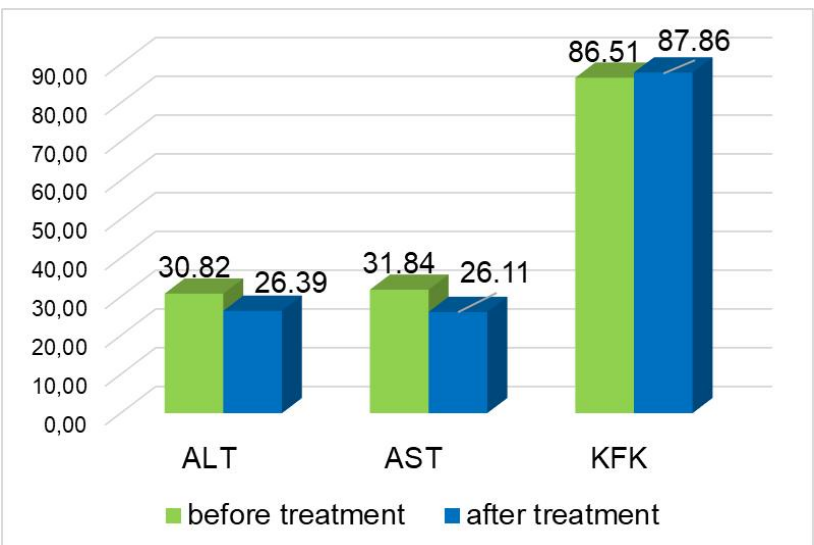

Fig. 3. Dynamics of biochemical parameters of myocardial destruction in patients who were prescribed intravesical doxorubicin.

heartbeat.

The third group of patients who received doxorubicin intravenously after surgery, as well as patients in the first group who did not receive chemotherapy felt well and had no symptoms of heart disease.

ECG data in the studied groups of patients are shown in table 1.
According to ECG data, in patients of the second group who received intravenous doxorubicin, the average heart rate increased from 68.11 to 75.03 . At the same time, the QRS complex expanded by $17.6 \%$. At the same time, no changes in ECG were observed in patients of the first and third groups.

The dynamics of changes in biochemical markers of myocardial destruction in the studied contingent is shown in Figures 1-3.

Determination of biochemical markers of myocardial destruction revealed changes in blood aminotransferases, namely, elevation of aspartate aminotransferase activity, was found in $43.7 \%$ of patients treated with intravenous doxorubicin. The average AST levels in such patients were $52.82 \mathrm{U} / \mathrm{l}$, which is 3.3 times higher than before treatment.

A similar trend was observed in the analysis of alanine aminotransferase (ALT) activity. Thus, the level of ALT after a course of intravenous doxorubicin increased 4.7 times and amounted to $71.86 \mathrm{U} / \mathrm{l}$ against $15.26 \mathrm{U} / \mathrm{l}$ before treatment.

At the same time, creatinine phosphokinase (CPK) activity did not differ significantly at the beginning and end of doxorubicin administration.

A completely different clinical picture was observed with intravesical doxorubicin. The activity of ALT, AST and CPK did not differ before and after the administration of the chemotherapeutic agent, and did not exceed the norm.

In the study of patients with bladder cancer who were treated only by surgery and did not receive doxorubicin by any of the above methods, AST activity also did not exceed normal and was 22.32 and $25.1 \mathrm{U} / \mathrm{l}$ before and after treatment, respectively, that was $17.02 \%$ less than the corresponding figure in the group of patients receiving doxorubicin systemically. It should be noted that at the end of treatment, these activity indicators did not differ statistically. A similar picture was observed in the analysis of the dynamics of changes in the levels of ALT and CPK.

\section{Discussion}

The use of doxorubicin has shown excellent results in the treatment of malignant tumors, but its wider use is hampered by its potential cardiotoxicity, which is clinically manifested by cardiomyopathy and congestive heart failure $[6,11,17]$.

The mechanism of cardiotoxicity of doxorubicin has not been fully studied. However, all anthracycline chemotherapeutics have some degree of cardiotoxic effect, which is due to: on the one hand - inhibition and destruction of topoisomerase IIB in cardiomyocytes, which leads to blocking DNA replication, and on the other hand - activation of oxidative stress in the heart and negative impact on repair processes, resulting in free anthracycline metabolites, increase apoptosis $[10,16]$. By penetrating cardiomyocytes, doxorubicin binds to proteasomes that transport the doxorubicin-proteasome complex to the nucleus, where it interacts with DNA double helices and a whole family of 
topoisomerase enzymes [12]. The depressant effect of doxorubicin on topoisomerase complexes leads to nonischemic irreversible cumulative cardiopathy, which is characterized by a number of symptoms, including: complaints of heart pain, arrhythmia, changes in ECG and ultrasound. According to the literature, signs of the effect of doxorubicin on the morphology of carbiomyocytes can be characterized by such changes as myocardial atrophy, fibrosis and disorganization of myocardial structure. At electron microscopy of hearts in experiments on micropreparations contracture and lysis of cardiomyocytes are noted. Muscle fibers are loose, sometimes destructured due to significant intermyofibrillar edema. Histological specimens also show changes in the side of the heart vessels, such as stagnation and redness in the venules and capillaries, perivascular edema and sometimes atherosclerotic changes in the vessels [5, 21].

Summarizing the results of a large number of diverse studies, it can be argued that oxidative stress, inflammation, apoptosis, mitochondrial dysfunction and calcium overload of calcium are the main components of the pathogenesis of toxic heart disease $[3,8,15,18,20]$.

Methods of preventing the development of cardiopathy with doxorubicin include replacement with drugs from other groups, which often reduces the effectiveness of treatment, concomitant use of beta blockers and calcium antagonists, this solution reduces symptoms but does not solve the problem of genetic aberrations caused by doxorubicin. The next method is the use of liposomal forms of doxorubicin, and this method of prevention, although effective, but this method is currently being studied and not widely used [19]. Given the low efficiency of modern methods of prevention of doxorubicin cardiopathy, the search for new methods of prevention of heart disease remains an urgent problem, one of which may be the local introduction of doxorubicin into the cavity to reduce its systemic damage to the heart.

\section{References}

[1] Babjuk, M., Burger, M., Comperat, E. M., Gontero, P., Mostafid, A. H., Palou, J., ... \& Soukup, V. (2019). European Association of Urology Guidelines on Non-muscle-invasive Bladder Cancer (TaT1 and Carcinoma In Situ) - 2019 Update. European urology, 76(5), 639-657. doi: 10.1016/j.eururo.2019.08.016

[2] De Angelis, R., Sant, M., Coleman, M. P., Francisci, S., Baili, P., Pierannunzio, D., ... \& EUROCARE-5 Working Group. (2014). Cancer survival in Europe 1999-2007 by country and age: results of EUROCARE--5-a population-based study. The Lancet. Oncology, 15(1), 23-34. doi: 10.1016/S14702045(13)70546-1

[3] Diamanti, J., Mezzetti, B., Giampieri, F., Alvarez-Suarez, J. M., Quiles, J. L., Gonzalez-Alonso, A., ... \& Battino, M. (2014). Doxorubicin-induced oxidative stress in rats is efficiently counteracted by dietary anthocyanin differently enriched strawberry (Fragaria - ananassa Duch.). Journal of agricultural and food chemistry, 62(18), 3935-3943. doi: 10.1021/jf405721d

[4] Ferlay, J., Steliarova-Foucher, E., Lortet-Tieulent, J., Rosso, S., Coebergh, J. W., Comber, H., ... \& Bray, F. (2013). Cancer incidence and mortality patterns in Europe: estimates for 40
Analyzing the results of the survey of patients in the study group, we found that doxorubicin when administered systemically causes deterioration of patients after the first year, as evidenced by the appearance of complaints of pain and heart failure. Toxic effects of doxorubicin were also confirmed in an instrumental study, which showed that $47 \%$ of patients showed tachycardia and dilatation of ventricular complexes. The appearance of the above complaints forced doctors to extend the intervals between courses to restore heart rate, and, consequently, worsened the prognosis for cure. Similar trends in the systemic use of doxorubicin have been described in the scientific literature $[6,11,17]$.

In contrast, doxorubicin therapy, when administered intravesically, did not cause complaints of cardiac function. This route of administration prevented the occurrence of cardiotoxicity in patients with bladder cancer, which is due to the almost complete absence of reabsorption of the drug from the bladder into the systemic bloodstream, and hence its accumulation in the heart.

In the group of patients who did not receive doxorubicin, we did not register any complaints and changes in both ECG results and biochemical markers of myocardial destruction.

Based on the above, in our opinion, serial intravesical use of doxorubicin can provide optimal treatment for bladder cancer.

\section{Conclusion}

1. Systemic serial administration of doxorubicin to patients with bladder cancer is accompanied by a low incidence of recurrence, but causes significantly significant changes in the myocardium after the first course of chemotherapy.

2. A single intravesical instillation of doxorubicin does not cause changes in laboratory and instrumental heart rate.

countries in 2012. European journal of cancer (Oxford, England : 1990), 49(6), 1374-1403. doi: 10.1016/ j.ejca.2012.12.027

[5] Kalyanaraman, B. (2020). Teaching the basics of the mechanism of doxorubicin-induced cardiotoxicity: Have we been barking up the wrong tree?. Redox biology, 29, 101394. doi: 10.1016/ j.redox.2019.101394

[6] Lehmann, L. H., \& Frohling, S. (2020). Mechanismen der Kardiotoxizitat onkologischer Therapien [Mechanisms of cardiotoxicity of oncological therapies]. Der Internist, 61(11), 1132-1139. doi: 10.1007/s00108-020-00881-2

[7] Machan, L., Burt, H. M., \& Hunter, W. L. (1997). Local delivery of chemotherapy: a supplement to existing cancer treatments. A case for surgical pastes and coated stents. Advanced drug delivery reviews, 26(2-3), 199-207. doi: 10.1016/s0169409x(97)00035-5

[8] Mandziuk, S., Gieroba, R., Korga, A., Matysiak, W., JodlowskaJedrych, B., Burdan, F., ... \& Dudka, J. (2015). The differential effects of green tea on dose-dependent doxorubicin toxicity. Food \& nutrition research, 59, 29754. doi: 10.3402/ fnr.v59.29754 
[9] Natsionalnyi instytut raku. (2020). Rak v Ukraini: Biuleten natsionalnoho kantser reiestru Ukrainy № 21 (21st ed.).

[10] Palmer, A., Mason, G. G., Paramio, J. M., Knecht, E., \& Rivett, A. J. (1994). Changes in proteasome localization during the cell cycle. European journal of cell biology, 64(1), 163-175.

[11] Pugazhendhi, A., Edison, T., Velmurugan, B. K., Jacob, J. A., \& Karuppusamy, I. (2018). Toxicity of Doxorubicin (Dox) to different experimental organ systems. Life sciences, 200, 26-30. doi: 10.1016/j.lfs.2018.03.023

[12] Rivett, A. J., Palmer, A., \& Knecht, E. (1992). Electron microscopic localization of the multicatalytic proteinase complex in rat liver and in cultured cells. The journal of histochemistry and cytochemistry : official journal of the Histochemistry Society, 40(8), 1165-1172. doi: 10.1177/40.8.1619280

[13] Schmidt, S., Kunath, F., Coles, B., Draeger, D. L., Krabbe, L. M., Dersch, R., ... \& Meerpohl, J. J. (2020). Intravesical Bacillus Calmette-Guerin versus mitomycin $\mathrm{C}$ for Ta and T1 bladder cancer. The Cochrane database of systematic reviews, 1(1), CD011935. doi: 10.1002/14651858.CD011935.pub2

[14] Siegel, R. L., Miller, K. D., \& Jemal, A. (2018). Cancer statistics, 2018. CA: a cancer journal for clinicians, 68(1), 7-30. doi: 10.3322/caac. 21442

[15] Sun, X. P., Wan, L. L., Yang, Q. J., Huo, Y., Han, Y. L., \& Guo, C. (2017). Scutellarin protects against doxorubicin-induced acute cardiotoxicity and regulates its accumulation in the heart. Archives of pharmacal research, 40(7), 875-883. doi: 10.1007/ s12272-017-0907-0

[16] Tan, C., Tasaka, H., Yu, K. P., Murphy, M. L., \& Karnofsky, D. A. (1967). Daunomycin, an antitumor antibiotic, in the treatment of neoplastic disease. Clinical evaluation with special reference to childhood leukemia. Cancer, 20(3), 333-353. doi: 10.1002/ 1097-0142(1967)20:3<333::aid-cncr2820200302>3.0.co;2-k

[17] van der Zanden, S. Y., Qiao, X., \& Neefjes, J. (2021). New insights into the activities and toxicities of the old anticancer drug doxorubicin. The FEBS journal, 288(21), 6095-6111. doi: 10.1111/febs. 15583

[18] Wenningmann, N., Knapp, M., Ande, A., Vaidya, T. R., \& AitOudhia, S. (2019). Insights into Doxorubicin-induced Cardiotoxicity: Molecular Mechanisms, Preventive Strategies, and Early Monitoring. Molecular pharmacology, 96(2), 219232. doi: $10.1124 / \mathrm{mol} .119 .115725$

[19] Xing, M., Yan, F., Yu, S., \& Shen, P. (2015). Efficacy and Cardiotoxicity of Liposomal Doxorubicin-Based Chemotherapy in Advanced Breast Cancer: A Meta-Analysis of Ten Randomized Controlled Trials. PloS one, 10(7), e0133569. doi: 10.1371/journal.pone.0133569

[20] Zhang, D., Yao, L., Yu, S., Cheng, Y., Jiang, J., Ma, Q., \& Yan, Z. (2020). Safety and efficacy of en bloc transurethral resection versus conventional transurethral resection for primary nonmuscle-invasive bladder cancer: a meta-analysis. World journal of surgical oncology, 18(1), 4. doi: 10.1186/ s12957-019-1776-4

[21] Zhao, L., \& Zhang, B. (2017). Doxorubicin induces cardiotoxicity through upregulation of death receptors mediated apoptosis in cardiomyocytes. Scientific reports, 7, 44735. doi: 10.1038/ srep44735

[22] Zhou, Z., Zhao, S., Lu, Y., Wu, J., Li, Y., Gao, Z., ... \& Cui, Y. (2019). Meta-analysis of efficacy and safety of continuous saline bladder irrigation compared with intravesical chemotherapy after transurethral resection of bladder tumors. World journal of urology, 37(6), 1075-1084. doi: 10.1007/ s00345-019-02628-7 